\title{
The Design of the Power Distribution Network Fault Location System Center Software
}

\author{
Zimeng Wang ${ }^{a}$, Yulin $\mathrm{Qi}^{\mathrm{b}}$, Jing Liu ${ }^{\mathrm{c}}$ and Zeli Qi ${ }^{\mathrm{d}}$ \\ School of Electronics and Communication Engineering, North China Electric Power University, \\ Baoding 071003, China \\ a1165667982@qq.com, 'oiyulin589@163.com, '951220813@qq.com, d526877160@qq.com
}

Keywords: Power distribution network, Fault location, the software design.

\begin{abstract}
Distribution network fault automatic location system is to solve the problem in the shortest possible time to find fault point, and the purpose is to develop a set of automatic and efficient fault point accurately addressing system. After the failure of a very short period of time, through the control center to give specific instructions, fault location and fault time inform the fault information to run maintenance personnel, in order to rapidly to the scene, troubleshooting, resume normal power supply, greatly improve the power supply reliability, at the same time greatly reduce fault patrol personnel, improve the work efficiency. The central station software, adopts the $\mathrm{C} / \mathrm{S}$ structure, using Visual $\mathrm{C}++$ as a development tool, database is the most popular used in the Access database management system, based on the distribution network line running status information acquisition, and real-time monitoring, to process the data received, judge the fault point and positioning, and accurate display on the software interface.
\end{abstract}

\section{Introduction}

Distribution network in electric power network plays an important role in allocation of electricity. However with the development of our country's economy, the scale of power system, gradually increase, the network structure gradually complicated, accident risk is bigger, corresponding once accidents, will directly affect the security and stability and economic operation of power system. Therefore, fast judgment fault location and troubleshooting is the premise to guarantee the safe and stable operation of power system [1,2]. To do this, we can not do without an energy management distribution network uptime, and can effectively and quickly deal with management software fault information [3]. Based on this viewpoint, this paper designed and developed a distribution network fault location system central station software designed to improve the efficiency and accuracy of diagnosis of distribution network, reduce blindness work with information technology for the modernization of the power grid to provide useful help.

\section{Software research contents and goals}

Central station software distribution network fault location system, through the distribution network line running status information acquisition, and real-time monitoring, to process the data received, judge the fault point and positioning, and accurate display on the central station software. System flow chart shown in figure 1, the software main research content is as follows:

2.1 Mapping the distribution network.

Since each installation site have different installation lines, we need to be installed according to different hardware line, trying to clear the distribution network circuit diagram displayed on the central station software interface that enables this figure reached visualization.

\subsection{Data acquisition and processing.}

Can capture footage back to the hardware real-time data, it is a prerequisite to handle after the data, so to be able to receive the correct data, and then analyze the data processing is an important part of this research. 


\subsection{Fault alarm.}

When data from judgment for the failure data, requirements will make alarm status, system software can occur through alarm inform staff fault location, and then to prepare for the repair.

The software contents you want to achieve the above objectives are: able to obtain through the central station software accurate data and analysis, and finally, accurate positioning distribution network failure points.

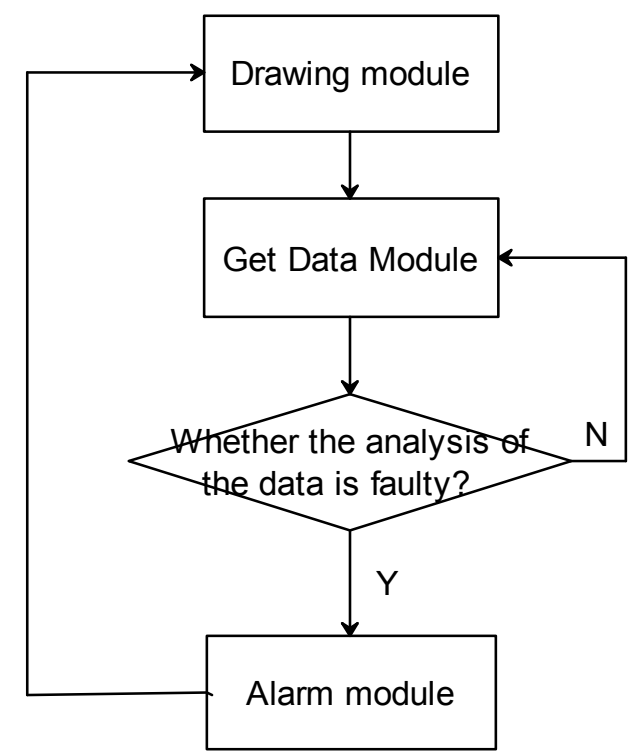

Fig. 1 The flow chart of system solutions

\section{Software Features}

According to system requirements, the results in the form of graphic interface reality to the user. Overall software interface diagram as shown in figure 2.

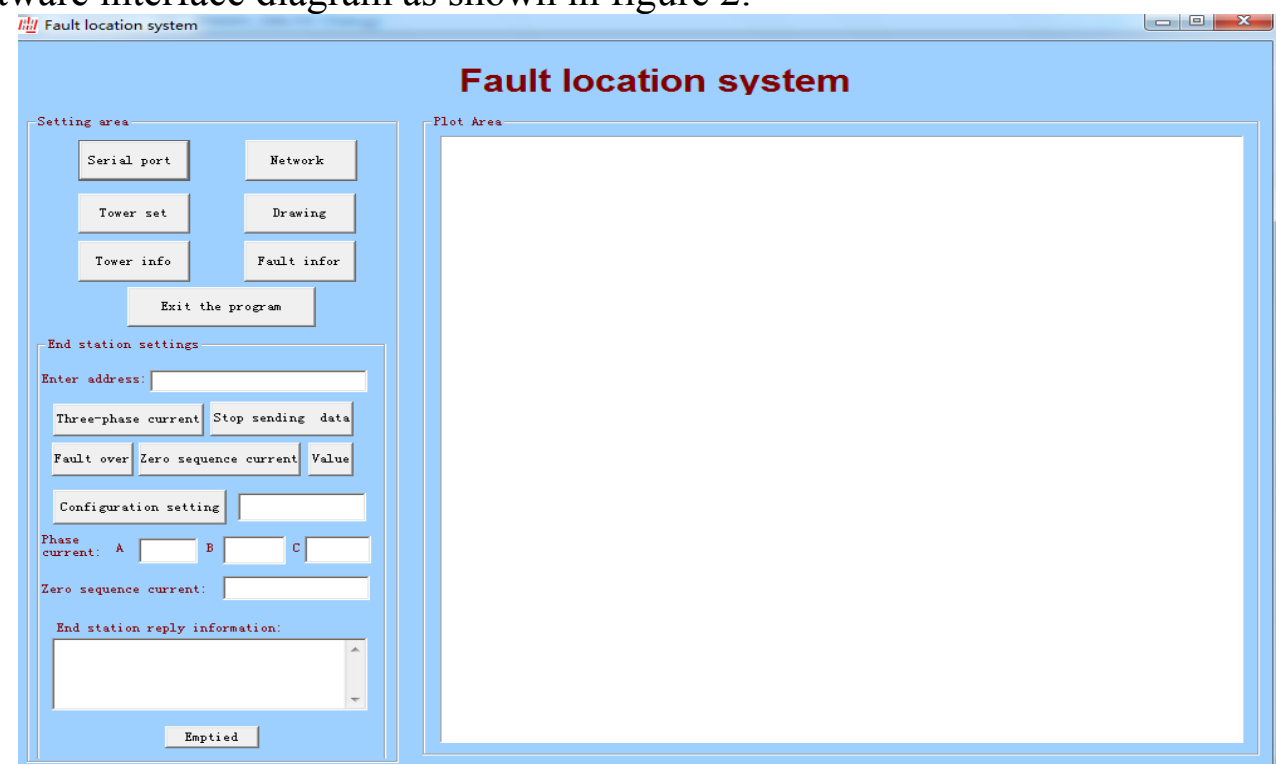

Fig. 2 Overall system interface diagram

This software will be divided into two functional areas, respectively is "set area" and "plot", which they implement specific function is as follows:

\section{1 "Settings" area function.}

Setting area includes a feature set region and seven function / command button, namely "end station setting area" and "Serial Port Settings" button, "Network Communication Settings" button, "tower Settings" button, "Drawing" button "tower info" button, "historical error message" button and "Exit" button, it is possible to achieve different functions, respectively. 
a. Serial port settings: Realize serial communication function set, including the use of communication serial port, baud rate, parity, data bits and stop bits, by default they COM1, 115200 respectively, NONE, 8 and 1, as shown in figure 3. These default Settings match the serial default Settings on your computer, usually don't need to change some Settings.

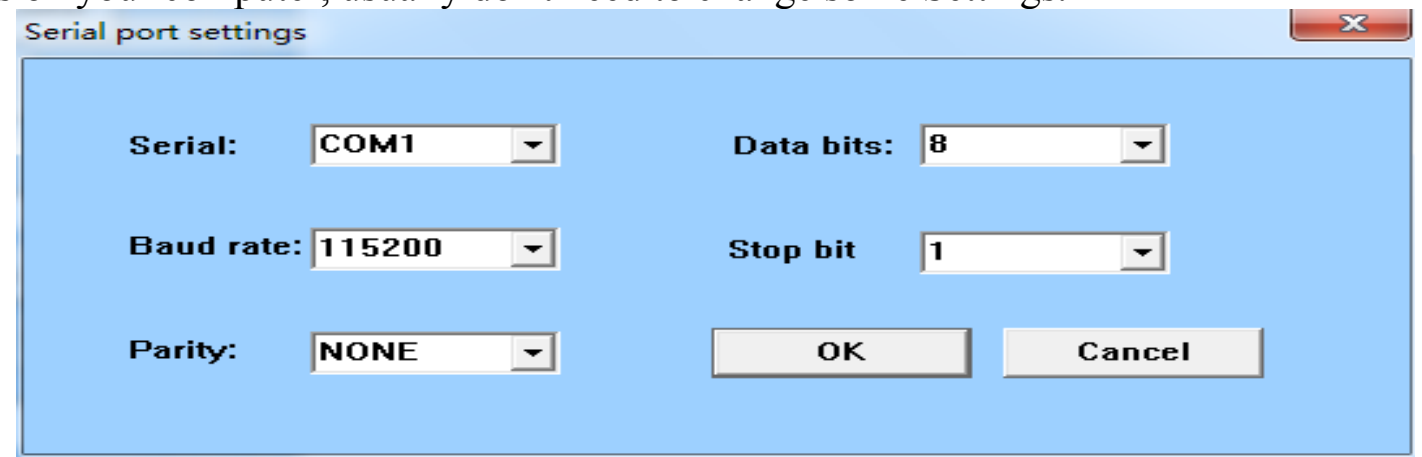

Fig. 3 Serial port settings

b. Network communication Settings: Network communication functions, via the built-in serial port BM100N centralized controller plus GPRS module configured fixed IP server and port number to connect to GPRS[4], automatically connect to the server via a fixed IP TCP / IP, and then send and receive data and commands. Enter the server a fixed IP address and port number to connect the server in Figure 4.

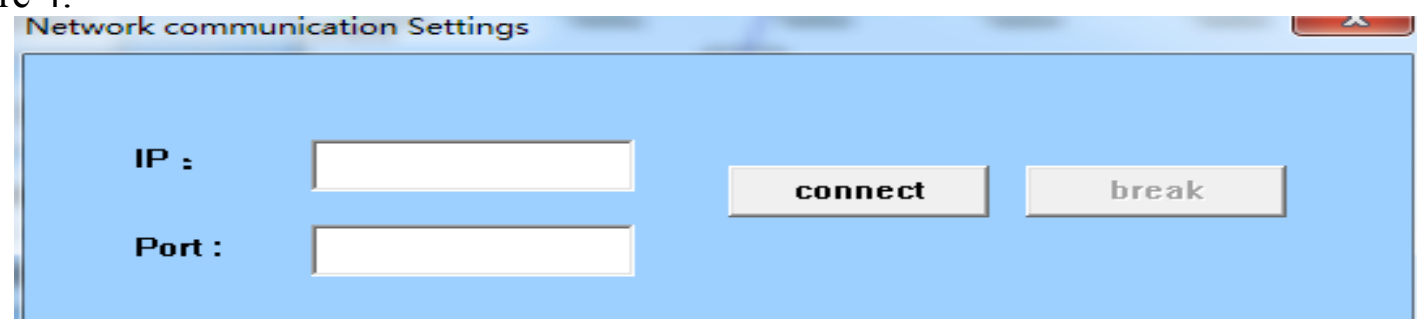

Fig. 4 Network communication Settings

c. Tower Set: Set up each tower-related information, including each tower number, tower address, substation number and line number belongs on a tower connected thereto tower number. This feature set is the basis for drawing, locate the fault occurred and alarm functions.

d. tower information: Achieve read the information on the tower. Administrators can read each tower tower number, tower address this feature, substation number and line number belongs.

e. Historical fault information:Management personnel can browse the past fault information through this function.

f. Drawing: According to information that has been set tower, tower distribution graph drawn under each line.

g. End station Settings area: Click on each tower by entering the address of the function button to get access to data in real-time three-phase current and zero sequence current data and restore data end station received for handling the fault lay the foundation for data analysis.

\section{2 "Plot Area" feature.}

This area can be based on information that has been set tower, visual display of the distribution of each line towers subordinates. At the same time when a failure occurs, the graphics can be distributed in the tower directly locate the position of the tower failed.

\section{Conclusion}

According to the basic current situation in China, the gradual increase in the size of the power system, the network structure became more complex, and that users of the stable power supply requirements are also getting higher and higher, the distribution network is a power system fault location in urgent problem [5]. Therefore, in order to more effectively locate the fault point, develop the distribution network fault location system central station software, accurately and quickly locate a specific point of failure, thus rapid troubleshooting. This design of "distribution network fault 
location system central station software" friendly interface, easy operation, practical features, specifically to achieve the following functions: simple, practical, and can quickly achieve tower towers fault information or add information, modify, or delete operation, greatly simplifying the operational processes, improve work efficiency; use ADO database access technology, the realization of intelligent data management, increasing the speed of information processing; The use of modern means of communication, to achieve the exchange of data center management software and tower between stations for fault alarm the foundation; Using GPRS module and serial communication technology, receive real-time fault information sent to the tower, and make timely alarm. The software in ensuring safe operation of the power system, improve power supply reliability is of great significance.

\section{References}

[1].Yuhui Guo, Yulin Qi. Based on the zero sequence current of single-phase grounding fault location system.Journal of electric power.Vol.27 (2012) No.6, p. 550-553.

[2].Wanshun Liu,Shaofeng Huang, Yuqin Xu. Power system fault analysis (third edition). China power press, 2010,p20-35.

[3].Feifei Zhu:Intelligent distribution network fault location research(MS., Yangzhou University, China 2012).p.2.

[4].Zhenhua Wu:The study of the distribution network fault location system based on GPRS (MS., Beijing Jiaotong University, China 2014).p.6.

[5]Yulin Qi: Research and implementation of single-phase grounding fault location of medium voltage distribution network(Dr.,North China Electric Power University, China 2007).p.2. 